E3S Web of Conferences 1, 40002 (2013)

DOI: $10.1051 / \mathrm{e} 3$ sconf/ 20130140002

(C) Owned by the authors, published by EDP Sciences, 2013

\title{
Arsenic and cardiovascular diseases
}

\author{
F. Bianchi ${ }^{1}$, L. Cori $^{1}$ and R. Sicari ${ }^{1}$ \\ ${ }^{1}$ Institute of Clinical Physiology, National Research Council, Pisa, Italy, fabrizio.bianchi@ifc.cnr.it, \\ liliana.cori@ifc.cnr.it, rosa.sicari@ifc.cnr.it
}

\begin{abstract}
A growing body of epidemiologic, experimental and clinical evidence shows that arsenic may exert relevant cardiovascular effects with early damage such as endothelial dysfunction. Early biomarkers of cardiovascular damage together with markers of exposure, genetic and epigenetic effects, DNA damage, apoptosis, oxidative stress remain unexplored and a study is ongoing in Italy.
\end{abstract}

Keywords: cardiovascular diseases, arsenic, biomonitoring, early markers of cardiovascular effects

\section{Introduction}

Inorganic arsenic and arsenic compounds have been classified in Group 1 "carcinogenic to humans" by IARC (2004). Both short- and long-term exposure to arsenic can cause several health problems. The interest in cardiovascular effects of human exposures to arsenic is growing.

Since the '90, human exposure at high level of arsenic concentration was associated with increased incidence of ischemic heart disease and cardiovascular mortality (Chen et al., 1996).

Associations with hypertension, diabetes mellitus and sub-clinic vascular damages such as carotid atherosclerosis were also suggested, which may also accelerate the incidence of arsenic-mediated induction of cardiovascular disorders (States et al., 2009).

Chronic exposure to arsenic through drinking water or acutely through medical therapy for acute promyelocytic leukemia or poisoning (Mumford et al., 2007; Westervelt et al., 2001; Sanz et al., 2009) is associated with cardiovascular abnormalities (States al., 2009; Wade et al., 2009).

Epidemiological, in-vitro end in-vivo experimental studies are supporting the endothelial dysfunction effect of arsenic exposures.

These results, although obtained through indirect methods, are reinforced by in-vitro end in-vivo experimental studies supporting a potential vascular effect of arsenic exposure. These effects may be exerted through different cellular and molecular mechanisms, which remain unsettled and unexplored. Moreover, therapeutic interventions (e.g. vitamin C, B9 and B12, alpha-tocopherol, curcumin, resveratrol) have been suggested to prevent or slow the development of arsenicinduced organ toxicity and cardiovascular disorders.

\section{Discussion}

More studies are needed to clarify the mechanisms involved in the induction of arsenic mediated cardiovascular disorders in view of preventing hazardous exposures and developing novel therapies to protect the cardiovascular system.

A human biomonitoring study is in progress in Italy, on adult population samples recruited in two areas with high levels of arsenic due to natural geological contamination (Amiata, Tuscany and Viterbo province, Lazio) and two areas with presence of arsenic of industrial origin (Taranto, Apulia and Gela, Sicily).

For each subjects, other than markers of exposure, genetic and epigenetic effects, DNA damages, apoptosis, cell stress collected in urine and blood, early markers of cardiovascular risk are collected. The study protocol includes: ECG, carotid ultrasound imaging, intima media thickness measurement, echocardiography, particularly to quantify the calcification score index that considers aortic root sclerosis, aortic valve sclerosis, and mitral annulus calcification.

These results may provide novel insights on markers of cardiovascular arsenic-induced damage and design new therapeutic interventions with preventive strategies for public health. 


\section{Acknowledgements}

The authors thank the financial support from the Italian Ministry of Health (Project SEpiAs, CCM 2010).

\section{References}

Balakumar, P., and Kaur, J. (2009). Arsenic exposure and cardiovascular disorders: an overview. Cardiovasc Toxicol 9, 169-176.

Chen, C.J., Chiou, H.Y., Chiang, M.H., Lin, L.J., and Tai, T.Y. (1996). Dose-response relationship between ischemic heart disease mortality and long-term arsenic exposure. Arterioscler Thromb Vasc Biol 16, 504-510.

Chen, Y., Factor-Litvak, P., Howe, G.R., Graziano, J.H., Brandt-Rauf, P., Parvez, F., Van Geen, A., and Ahsan, H. (2007a). Arsenic exposure from drinking water, dietary intakes of B vitamins and folate, and risk of high blood pressure in Bangladesh: a population-based, cross-sectional study. Am J Epidemiol 165, 541-552.

Chen, Y., Parvez, F., Gamble, M., Islam, T., Ahmed, A., Argos, M., Graziano, J.H., and Ahsan, H. (2009). Arsenic exposure at low-to-moderate levels and skin lesions, arsenic metabolism, neurological functions, and biomarkers for respiratory and cardiovascular diseases: review of recent findings from the Health
Effects of Arsenic Longitudinal Study (HEALS) in Bangladesh. Toxicol Appl Pharmacol 239, 184-192.

Chen, Y., Santella, R.M., Kibriya, M.G., Wang, Q., Kappil, M., Verret, W.J., Graziano, J.H., and Ahsan, H. (2007b). Association between arsenic exposure from drinking water and plasma levels of soluble cell adhesion molecules. Environ Health Perspect 115, 1415-1420.

Lee, M.Y., Jung, B.I., Chung, S.M., Bae, O.N., Lee, J.Y., Park, J.D., Yang, J.S., Lee, H., and Chung, J.H. (2003). Arsenic-induced dysfunction in relaxation of blood vessels. Environ Health Perspect 111, 513517.

Pi, J., Kumagai, Y., Sun, G., Yamauchi, H., Yoshida, T., Iso, H., Endo, A., Yu, L., Yuki, K., Miyauchi, T., and Shimojo, N. (2000). Decreased serum concentrations of nitric oxide metabolites among Chinese in an endemic area of chronic arsenic poisoning in inner Mongolia. Free Radic Biol Med 28, 1137-1142.

States, J.C., Srivastava, S., Chen, Y., and Barchowsky, A. (2009). Arsenic and cardiovascular disease. Toxicol Sci 107, 312-323.

Wu, M.M., Chiou, H.Y., Ho, I.C., Chen, C.J., and Lee, T.C. (2003). Gene expression of inflammatory molecules in circulating lymphocytes from arsenicexposed human subjects. Environ Health Perspect $111,1429-1438$. 